\title{
Global and local behavior of a class of $\xi^{(s)}$ QSSO
}

\author{
Abdelwahab Alsarayreh $^{\mathrm{a}, *}$, Izzat Qaralleh ${ }^{\mathrm{b}}$, Muhammad Zaini Ahmad ${ }^{\mathrm{a}}$, Basma Al-Shutnawi ${ }^{\mathrm{b}}$, Saba $^{\mathrm{b}}$ \\ Al-Kaseasbeh ${ }^{b}$ \\ a Institute of Engineering Mathematics, University Malaysia Perlis Pauh Putra Main Campus, 02600 Arau, Perlis, Malaysia. \\ ${ }^{b}$ Department of mathematics, Faculty of Science, Tafila Technical University, P. O. Box, 179, 66110, Tafila, Jordan.
}

Communicated by B. Samet

\begin{abstract}
A quadratic stochastic operator (QSO) describes the time evolution of different species in biology. The main problem with regard to a nonlinear operator is to study its behavior. This has not been studied in depth; even QSOs, which are the simplest nonlinear operators, have not been studied thoroughly. This paper investigates the global behavior of an operator taken from $\xi^{(s)}$-QSO when the parameter $a=\frac{1}{2}$. Moreover, we study the local behavior of this operator at each value of $a$, where $0<a<1$. (C)2017 All rights reserved.
\end{abstract}

Keywords: Quadratic stochastic operator, local behavior, global behavior. 2010 MSC: 37E99, 37N25, 39B82, 47H60, 92D25.

\section{Introduction}

The history of quadratic stochastic operators (QSOs) can be traced back to Bernstein's work [1]. The QSO was considered an important source of analysis for the study of dynamical properties and modelings in various fields such as biology [1, 14, 15, 18-20, 34], physics [25, 32], economics, and mathematics $[15,17,20,33]$.

One such system related to population genetics is given by a QSO [1], which is commonly used to present the time evolution of species in biology, which arises as follows: consider a population that consists of $m$ species (or traits) $1,2, \cdots, m$. We denote a set of all species (traits) by $I=\{1,2, \cdots, m\}$. Let $\chi^{(0)}=\left(x_{1}^{(0)}, \cdots, x_{\mathrm{m}}^{(0)}\right)$ be a probability distribution of species at an initial state and $\mathrm{P}_{i j, \mathrm{k}}$ be a probability that individuals in the $i^{\text {th }}$ and $j^{\text {th }}$ species (traits) interbreed to produce an individual from $k^{\text {th }}$ species (trait). Then a probability distribution $x^{(1)}=\left(x_{1}^{(1)}, \ldots, x_{\mathrm{m}}^{(1)}\right)$ of the spices (traits) in the first generation can be found as a total probability, i.e.,

$$
x_{k}^{(1)}=\sum_{i, j=1}^{m} P_{i j, k} x_{i}^{(0)} x_{j}^{(0)}, \quad k=\overline{1, m} .
$$

\footnotetext{
*Corresponding author

Email addresses: abdalwahb.saraereh@gmail.com (Abdelwahab Alsarayreh), izzat_math@yahoo.com (Izzat Qaralleh), mzaini@unimap.edu.my (Muhammad Zaini Ahmad), salmashut@yahoo.com (Basma Al-Shutnawi), skasabeh@ttu.edu.jo (Saba Al-Kaseasbeh) 
This result means that the association $x^{(0)} \rightarrow x^{(1)}$ defines a mapping $V$ called the evolution operator. The population evolves by starting from an arbitrary state $x^{(0)}$, then passing to the state $x^{(1)}=V\left(x^{(0)}\right)$ (the first generation), then to the state $x^{(2)}=\mathrm{V}\left(x^{(1)}\right)=\mathrm{V}\left(\mathrm{V}\left(x^{(0)}\right)\right)=\mathrm{V}^{(2)}\left(x^{(0)}\right)$ (the second generation), and so on. Therefore, the evolution states of the population system are described by the following discrete dynamical system:

$$
x^{(0)}, \quad x^{(1)}=\mathrm{V}\left(x^{(0)}\right), \quad x^{(2)}=\mathrm{V}^{(2)}\left(x^{(0)}\right), \quad x^{(3)}=\mathrm{V}^{(3)}\left(x^{(0)}\right), \cdots
$$

In other words, a QSO describes a distribution of the next generation if the distribution of the current generation was given. The fascinating applications of QSO to population genetics were given in [20].

A self-contained exposition of the recent achievements and open problems in the theory of QSO were given in [11]. The main problem in nonlinear operator theory is to study the behavior of nonlinear operators. This problem was not fully addressed even in the class of QSOs, which is the simplest nonlinear operator. The difficulty of the problem depends on the given cubic matrix $\left(P_{i j k}\right)_{i, j, k=1}^{m}$. An asymptotic behavior of the QSO even on the small dimensional simplex is complicated [4,30,31, 33, 35]. To solve this problem, many researchers always introduced a certain class of QSOs and studied their behavior. For examples, Volterra-QSO [5, 6, 10, 16, 33], permutated Volterra-QSO [8, 9], Quasi-Volterra-QSO [12], $\ell$-Volterra-QSO [27, 28], non-Volterra-QSO [4, 31], strictly non-Volterra-QSO [36], F-QSO [29], and nonVolterra operators generated by product measure $[7,13,26]$. However, all these classes together would not cover a set of all QSOs. Therefore, many classes of QSOs have not been studied yet. Recently, a new class of QSOs called $\xi^{(s)}$-QSO was introduced in $[21,22,24]$. In this paper, we will continue the study of $\xi^{(s)}$-QSO. This class of operators depends on a partition of the coupled index set (the coupled trait set) $\mathbf{P}_{m}=\{(i, j): i<j\} \subset I \times I$. In the case of two-dimensional simplex $(m=3)$, the coupled index set (the coupled trait set) $\mathbf{P}_{3}$ has five possible partitions. The dynamics of $\xi^{(s)}-Q S O$ that correspond to the point partition (the maximal partition) of $\mathbf{P}_{3}$ have been investigated in [21-24].

The paper is organized as follows. In Section 2, we present some preliminary definitions. In Section 3 , we find the fixed points of $V_{a}$. In Section 4 , we study the global behavior of $V_{\frac{1}{2}}$. In Section 5 , we investigate the local behavior of $\mathrm{V}_{\mathrm{a}}$.

\section{Preliminaries}

Definition 2.1. QSO is a mapping of the simplex

$$
S^{m-1}=\left\{x=\left(x_{1}, \cdots, x_{m}\right) \in \mathbb{R}^{m}: \sum_{i=1}^{m} x_{i}=1, \quad x_{i} \geqslant 0, \quad i=\overline{1, m}\right\}
$$

into itself of the form

$$
x_{k}^{\prime}=\sum_{i, j=1}^{m} P_{i j, k} x_{i} x_{j}, \quad k=\overline{1, m}
$$

where $V(x)=x^{\prime}=\left(x_{1}^{\prime}, \cdots, x_{m}^{\prime}\right)$ and $P_{i j, k}$ is a coefficient of heredity, which satisfies the following conditions

$$
P_{i j, k} \geqslant 0, \quad P_{i j, k}=P_{j i, k}, \quad \sum_{k=1}^{m} P_{i j, k}=1 .
$$

From the above definition we can conclude that each QSO $V: S^{m-1} \rightarrow S^{m-1}$ can be uniquely defined by a cubic matrix $\mathcal{P}=\left(\mathrm{P}_{i j k}\right)_{i, j, k=1}^{m}$ with conditions (2.2).

We will denote by Fix $(V)$ the set of fixed points of $V: S^{m-1} \rightarrow S^{m-1}$. Given Brouwer's fixed point theorem, one has always $\operatorname{Fix}(V) \neq \emptyset$ for any QSO $V$. For a given point $x^{(0)} \in S^{m-1}$, a trajectory $\left\{x^{(\mathfrak{n})}\right\}_{\mathfrak{n}=0}^{\infty}$ 
of $V: S^{m-1} \rightarrow S^{m-1}$ starting from $x^{(0)}$ is defined by $x^{(n+1)}=V\left(x^{(n)}\right)$. By $\omega_{V}\left(x^{(0)}\right)$, we denote a set of omega limiting points of the trajectory $\left\{x^{(n)}\right\}_{\mathfrak{n}=0}^{\infty} .\left\{x^{(n)}\right\}_{\mathfrak{n}=0}^{\infty} \subset S^{m-1}$ and $S^{m-1}$ are compact. Thus, one has $\omega_{V}\left(\chi^{(0)}\right) \neq \emptyset$. Obviously, if $\omega_{V}\left(\chi^{(0)}\right)$ consists of a single point, then the trajectory converges, and a limiting point is a fixed point of $\mathrm{V}: \mathrm{S}^{\mathrm{m}-1} \rightarrow \mathrm{S}^{\mathrm{m}-1}$.

Recall that a Volterra-QSO is defined by (2.1), (2.2), and the additional assumption

$$
P_{i j, k}=0 \quad \text { if } k \notin\{i, j\} .
$$

The biological treatment of condition (2.3) is clear: the offspring repeats the genotype (trait) of one of its parents.

One can see that a Volterra-QSO has the following form:

$$
x_{k}^{\prime}=x_{k}\left(1+\sum_{i=1}^{m} a_{k i} x_{i}\right), k \in I,
$$

where

Moreover,

$$
a_{k i}=2 P_{i k, k}-1 \text { for } i \neq k \text { and } a_{i i}=0, i \in I \text {. }
$$

$$
a_{k i}=-a_{i k} \text { and }\left|a_{k i}\right| \leqslant 1 \text {. }
$$

This type of operator was intensively studied in $[2,5,6,10,16,33]$. Note that this operator is a discretization of the Lotka-Volterra model $[19,34]$ which models an interacting competing species in the population system. Such a model has received considerable attention in the fields of biology, economy, and mathematics (see for example [14, 15, 25, 34]).

The concept of $\ell$-Volterra-QSO, which generalizes a notion of Volterra-QSO, was introduced in [27]. This concept is recalled as follows.

In order to introduce a new class of QSOs, we need some auxiliary notations. We fix $\ell \in \mathrm{I}$ and assume that elements $P_{i j, k}$ of the matrix $\left(P_{i j, k}\right)_{i, j, k=1}^{m}$ satisfy

$$
\begin{gathered}
P_{i j, k}=0 \text { if } k \notin\{i, j\} \text { for any } k \in\{1, \ldots, \ell\}, i, j \in I, \\
P_{i_{0} j_{0}, k}>0 \text { for some }\left(i_{0}, j_{0}\right), i_{0} \neq k, j_{0} \neq k, k \in\{\ell+1, \ldots, m\} .
\end{gathered}
$$

For any fixed $\ell \in \mathrm{I}$, the QSO defined by (2.1), (2.2), (2.4), and (2.5) is called $\ell$-Volterra-QSO.

Remark 2.2. Here, we emphasize the following points.

1. An $\ell$-Volterra-QSO is a Volterra-QSO if and only if $\ell=\mathrm{m}$.

2. No periodic trajectory exists for Volterra-QSO [5]. However, such trajectories exist for $\ell$-VolterraQSO [27].

By following [28], take $k \in\{1, \ldots, \ell\}$, then $P_{k k, i}=0$ for $i \neq k$ and

$$
1=\sum_{i=1}^{m} P_{k k, i}=P_{k k, k}+\sum_{i=\ell+1}^{m} P_{k k, i} .
$$

With the use of $P_{i j, k}=P_{j i, k}$ and denoting $a_{k i}=2 P_{i k, k}-1, k \neq i, a_{k k}=P_{k k, k}-1$ one then obtains

$$
V:= \begin{cases}x_{k}^{\prime}=x_{k}\left(1+\sum_{i=1}^{m} a_{k i} x_{i}\right), & \text { if } k=\overline{1, \ell}, \\ x_{k}^{\prime}=x_{k}\left(1+\sum_{i=1}^{m} a_{k i} x_{i}\right)+\sum_{\substack{i, j=1 \\ i \neq k, j \neq k}}^{m} P_{i j, k} x_{i} x_{j}, & \text { if } k=\overline{\ell+1, m .}\end{cases}
$$

This is a canonical form of $\ell$-Volterra-QSO.

Note that

$$
a_{k k} \in[-1,0],\left|a_{k i}\right| \leqslant 1, a_{k i}+a_{i k}=2\left(P_{i k, i}+P_{i k, k}\right)-2 \leqslant 0, i, k \in I \text {. }
$$

We recall that an operator $\mathrm{V}$ is permuted $\ell$-Volterra-QSO, if there is a permutation $\tau$ of the set $\mathrm{I}$ and an $\ell$-Volterra-QSO $\mathrm{V}_{0}$ such that $(\mathrm{V}(\mathrm{x}))_{\tau(\mathrm{k})}=\left(\mathrm{V}_{0}(\mathrm{x})\right)_{\mathrm{k}}$ for any $\mathrm{k} \in \mathrm{I}$. In other words, $\mathrm{V}$ can be represented as 
follows:

$$
V_{\tau}:= \begin{cases}x_{\tau(k)}^{\prime}=x_{k}\left(1+\sum_{i=1}^{m} a_{k i} x_{i}\right), & \text { if } k=\overline{1, \ell}, \\ x_{\tau(k)}^{\prime}=x_{k}\left(1+\sum_{i=1}^{m} a_{k i} x_{i}\right)+\sum_{\substack{i, j=1 \\ i \neq k, j \neq k}}^{m} P_{i j, k} x_{i} x_{j}, & \text { if } k=\overline{\ell+1, m .}\end{cases}
$$

By following [24], each element $x \in S^{m-1}$ is a probability distribution of the set $I=\{1, \ldots, m\}$. Let $x=\left(x_{1}, \cdots, x_{m}\right)$ and $y=\left(y_{1}, \cdots, y_{m}\right)$ be vectors taken from $S^{m-1}$. We say that $x$ is equivalent to $y$ if $x_{k}=0 \Leftrightarrow y_{k}=0$. We denote this relation by $x \sim y$.

Let $\operatorname{supp}(x)=\left\{i: x_{i} \neq 0\right\}$ be a support of $x \in S^{m-1}$. We say that $x$ is singular to $y$ and denote by $x \perp y$, if $\operatorname{supp}(x) \cap \operatorname{supp}(y)=\emptyset$. Note that if $x, y \in S^{m-1}$ then $x \perp y$ if and only if $(x, y)=0$, where $(\cdot, \cdot)$ stands for a standard inner product in $\mathbb{R}^{\mathrm{m}}$.

We denote sets of coupled indices by

$$
\mathbf{P}_{\mathrm{m}}=\{(\boldsymbol{i}, \mathbf{j}): \mathbf{i}<\mathbf{j}\} \subset \mathrm{I} \times \mathrm{I}, \quad \Delta_{\mathrm{m}}=\{(\mathfrak{i}, \mathrm{i}): \mathfrak{i} \in \mathrm{I}\} \subset \mathrm{I} \times \mathrm{I} .
$$

For a given pair $(i, j) \in \mathbf{P}_{m} \cup \Delta_{m}$, we set a vector $\mathbb{P}_{i j}=\left(P_{i j, 1}, \cdots, P_{i j, m}\right)$. Clearly, because of condition (2.2), $\mathbb{P}_{i j} \in S^{m-1}$.

Let $\xi_{1}=\left\{A_{i}\right\}_{i=1}^{N}$ and $\xi_{2}=\left\{B_{i}\right\}_{i=1}^{M}$ be some fixed partitions of $\mathbf{P}_{m}$ and $\Delta_{m}$, respectively, i.e. $A_{i} \cap A_{j}=\emptyset$, $B_{i} \cap B_{j}=\emptyset$, and $\bigcup_{i=1}^{N} A_{i}=P_{m}, \bigcup_{i=1}^{M} B_{i}=\Delta_{m}$, where $N, M \leqslant m$.

Definition 2.3 ([24]). QSO V : $S^{m-1} \rightarrow S^{m-1}$ given by (2.1), (2.2), is called a $\xi^{(\text {as) }}$-QSO w.r.t. the partitions $\xi_{1}, \xi_{2}$ (where the "as" stands for absolutely continuous-singular) if the following conditions are satisfied:

(i) for each $k \in\{1, \ldots, N\}$ and any $(i, j),(u, v) \in A_{k}$, one has $\mathbb{P}_{i j} \sim \mathbb{P}_{u v}$;

(ii) for any $k \neq \ell, k, \ell \in\{1, \ldots, N\}$ and any $(i, j) \in A_{k}$ and $(u, v) \in A_{\ell}$ one has $\mathbb{P}_{i j} \perp \mathbb{P}_{u v}$;

(iii) for each $d \in\{1, \ldots, M\}$ and any $(i, i),(j, j) \in B_{d}$, one has $\mathbb{P}_{i i} \sim \mathbb{P}_{j j}$;

(iv) for any $s \neq h, s, h \in\{1, \ldots, M\}$ and any $(u, u) \in B_{s}$ and $(v, v) \in B_{h}$, one has that $\mathbb{P}_{u u} \perp \mathbb{P}_{v v}$.

Definition $2.4([3])$. A fixed point $\bar{x}$ of the nonlinear system $x_{t+1}=\phi\left(x_{t}\right)$ is:

(i) globally (asymptotically) stable, if

$$
\lim _{\mathrm{t} \rightarrow \infty} x_{\mathrm{t}}=\bar{x} \quad \forall x_{0} \in \mathbb{R}^{\mathrm{n}} ;
$$

(ii) locally (asymptotically) stable, if there exist $\epsilon>0$, such that

$$
\lim _{\mathrm{t} \rightarrow \infty} x_{\mathrm{t}}=\overline{\mathrm{x}}, \quad \forall \mathrm{x}_{0} \in \mathrm{B}_{\epsilon}(\overline{\mathrm{x}}),
$$

where

$$
\mathrm{B}_{\epsilon}(\bar{x})=\left\{x \in \mathbb{R}^{n}:\left|x_{i}-\overline{x_{i}}\right|<\epsilon, \quad \forall i=1,2,3, \cdots, n\right\} .
$$

In this paper, we are going to investigate the global behavior of $V_{\frac{1}{2}}$ and the local behavior of $V_{a}$ which belongs to $\ell$-Volterra-QSO.

\section{Fixed points of $V_{a}$}

In [24], it has been classified the $\xi^{(s)}$-QSO into 20 non-conjugate classes. In this paper we are going to continue study the behavior of these classes, one of them is $K_{7}=\left\{V_{7}\right\}$, the operator $V_{7}$ which can be written by Tables 1 and 2 . 
Table 1: Coefficient of $\mathbf{P}_{3}$.

\begin{tabular}{llll}
\hline case & $P_{12}$ & $P_{13}$ & $P_{23}$ \\
\hline$I_{2}$ & $(0, a, 1-a)$ & $(0, a, 1-a)$ & $(1,0,0)$ \\
\hline
\end{tabular}

Table 2: Coefficient of $\Delta_{3}$.

\begin{tabular}{llll}
\hline case & $P_{11}$ & $P_{22}$ & $P_{33}$ \\
\hline $\mathrm{II}_{1}$ & $(1,0,0)$ & $(0,1,0)$ & $(0,0,1)$
\end{tabular}

By combination of Tables 1 and 2 , we get $V_{7}$. In this paper we will denote $V_{7}$ by $V_{a}$. Moreover, in this section we will find the fixed point of $\mathrm{V}_{\mathrm{a}}$,

$$
v_{a}:=\left\{\begin{array}{l}
x^{\prime}=x^{2}+2 y z \\
y^{\prime}=y^{2}+2 a x(1-x) \\
z^{\prime}=z^{2}+2(1-a) x(1-x)
\end{array}\right.
$$

where $0 \leqslant a \leqslant 1$. This operator is an $\ell$-Volterra-QSO. Let $e_{1}, e_{2}, e_{3}$ be the vertices of the simplex $S^{2}$.

Theorem 3.1. Let $\mathrm{V}_{\mathrm{a}}: \mathrm{S}^{2} \rightarrow \mathrm{S}^{2}$ be a $\xi^{(\mathrm{s})}-Q S O$ given by (3.1). Then

$$
\operatorname{Fix}\left(V_{a}\right)= \begin{cases}\left\{e_{2}, e_{3}\right\}, & \text { if } 0 \leqslant a \leqslant 1, \\ \left\{e_{1},\left(\frac{1}{3}, \frac{1}{3}, \frac{1}{3}\right)\right\}, & \text { if } a=\frac{1}{2}, \\ \left\{\left(x^{*}, y^{*}, z^{*}\right)\right\}, & \text { if } a \in\left(\frac{1}{4}, \frac{1}{2}\right) \cup\left(\frac{1}{2}, \frac{3}{4}\right),\end{cases}
$$

where $x^{*}=\frac{16 a^{2}-16 a+3}{16 a^{2}-16 a+1}, y^{*}=\frac{1-4 a}{16 a^{2}-16 a+1}$, and $z^{*}=1-x^{*}-y^{*}$.

Proof. To find the fixed points of (3.1), we should solve the following system of equations:

$$
\left\{\begin{array}{l}
x=x^{2}+2 y z \\
y=y^{2}+2 a x(1-x) \\
z=z^{2}+2(1-a) x(1-x)
\end{array}\right.
$$

Now, we shall separately consider two cases. Namely, $a=\frac{1}{2}$ and $a \neq \frac{1}{2}$.

Let $a=\frac{1}{2}$. Then, by subtracting third equation from second equation in (3.2) we obtain $y-z=$ $y^{2}-z^{2}$. If $y \neq z$ then $y+z=1$, therefore, $x=0$. It follows that $y^{2}=y$ and $z^{2}=z$. By solving these quadratic equations we have a fixed points $\left\{e_{2}, e_{3}\right\}$. Consider now $y=z$. From the first equation of (3.2) and $x+y+z=1$ we obtain $y=\frac{1}{2}(1-x)$. Hence, $x^{2}+2\left(\frac{1-x}{2}\right)^{2}=x$. Solving the last quadratic equation obtains $x \in\left\{1, \frac{1}{3}\right\}$. Thus we obtain fixed points $\left\{e_{1},\left(\frac{1}{3}, \frac{1}{3}, \frac{1}{3}\right)\right\}$.

Assume $a \neq \frac{1}{2}$. Replacing $z$ by $1-x-y$ in equation (1) of (3.2) we can write (1) as

$$
x^{2}+2 y-2 y x-2 y^{2}-x=0
$$

Rewriting the second equation of (3.2) gives

$$
y^{2}+2 a x-2 a x^{2}-y=0 .
$$

By adding (3.3) to (3.4), we obtain the following equation

$$
x((1-4 a) x+(4 a-1)-2 y)=0 .
$$


The solutions of equation (3.5) are $\left\{0, \frac{2 y-4 a+1}{1-4 a}\right\}$. If $x=0$, then it follows from equation (3.3) that $y^{2}-y=0$. Therefore, $y \in\{0,1\}$. Then we have the fixed points $\left\{e_{2}, e_{3}\right\}$. If $x=\frac{2 y-4 a+1}{1-4 a}$ then $y=\frac{1}{2}(x-1)(1-4 a)$. By inserting this value in first equation of (3.2) we obtain

$$
(x-1)\left(x+\frac{1}{2}(x-1)(1-4 a)(-3+4 a)\right)=0 .
$$

So, $x \in\left\{1, \frac{16 a^{2}-16 a+3}{16 a^{2}-16 a+1}\right\}$. If $x=1$, then we have the fixed point $e_{1}$. If $x=x^{*}$ then one obtains point $x^{*}=\frac{16 a(a-1)+3}{16 a(a-1)+1}, y^{*}=\frac{1-4 a}{16 a(a-1)+1}$ and $z^{*}=1-x^{*}-y^{*}$. We are now in the position to find out the conditions of the parameter a that makes the last fixed point lies in the simplex. To achieve this goal, we have to use the $0<\frac{x+3}{x+1}<1$ whenever $x \in\left[\frac{1}{3}, \frac{3}{4}\right]$

Therefore, if $a \in\left[\frac{1}{3}, \frac{3}{4}\right]$, then we obtain the fixed point $\left(x^{*}, y^{*}, z^{*}\right)$. If $a=\frac{1}{3}$ one has the fixed point $e_{2}$, and if $a=\frac{1}{2}$, then we obtain the fixed point $\left(\frac{1}{3}, \frac{1}{3}, \frac{1}{3}\right)$. The value of $a=\frac{3}{4}$ gives the fixed point $e_{3}$. This process completes the proof.

\section{Global behavior of $V_{\frac{1}{2}}$}

In this section we will study the global behavior of $V_{a}$ when $a=\frac{1}{2}$. Moreover, let us define the following regions:

$$
\begin{aligned}
& A_{1}:=\left\{(x, y, z) \in S^{2}: 0 \leqslant x, y, z \leqslant \frac{1}{2}\right\}, \\
& A_{2}:=\left\{(x, y, z) \in S^{2}: \frac{1}{2} \leqslant x<1\right\}, \\
& A_{3}:=\left\{(x, y, z) \in S^{2}: \frac{1}{2} \leqslant y<1\right\}, \\
& B_{1}:=\left\{(x, y, z) \in S^{2}: 0 \leqslant x, y, z \leqslant \frac{1}{2}, \quad x \leqslant z\right\}, \\
& B_{2}:=\left\{(x, y, z) \in S^{2}: 0 \leqslant x, y, z \leqslant \frac{1}{2}, \quad z \leqslant x\right\}, \\
& B_{3}:=\left\{(x, y, z) \in S^{2}: 0 \leqslant x, y, z \leqslant \frac{1}{2}, \quad y \leqslant x\right\}, \\
& B_{4}:=\left\{(x, y, z) \in S^{2}: 0 \leqslant x, y, z \leqslant \frac{1}{2}, \quad \frac{1}{3} \leqslant x \leqslant z\right\}, \\
& B_{5}:=\left\{(x, y, z) \in S^{2}: 0 \leqslant x, y, z \leqslant \frac{1}{2}, \quad 0 \leqslant z \leqslant x \leqslant \frac{1}{3} \leqslant y \leqslant \frac{1}{2}\right\} .
\end{aligned}
$$

Proposition 4.1. The following statements hold for $\mathrm{V}_{\frac{1}{2}}$ :

(1) the region $A_{1}$ is invariant;

(2) if $x^{(0)} \notin \operatorname{Fix}\left(V_{\frac{1}{2}}\right)$, and $x^{(0)} \in A_{2}$, then, $V^{(n)}\left(x^{(0)}\right)$ goes to $A_{1} \cup A_{3}$;

(3) if $x^{(0)} \notin \operatorname{Fix}\left(V_{\frac{1}{2}}\right)$, and $x^{(0)} \in A_{3}$, then, $V^{(n)}\left(x^{(0)}\right)$ goes to $A_{1}$.

Proof.

(1). Let $x^{(0)}=(x, y, z) \in A_{1}$, then $0 \leqslant x, y, z \leqslant \frac{1}{2}$. We want to show that $0 \leqslant x^{\prime}, y^{\prime}, z^{\prime} \leqslant \frac{1}{2}$. To achieve this objective, we can easily show that $-1 \leqslant 3 x-1 \leqslant \frac{1}{2}$ by squaring the last inequality, we obtain $0 \leqslant$ $(3 x-1)^{2} \leqslant 1$. Given $(y-z)^{2} \geqslant 0$, it follows that $(3 x-1)^{2}-3(y-z)^{2} \leqslant 1$. Thus, $9 x^{2}-6 x+1-3(y-z)^{2} \leqslant 1$. Adding 2 for both sides and dividing the resulting inequality by 3 , one finds that

$$
3 x^{2}-2 x+1-(y-z)^{2} \leqslant 1 .
$$


Therefore,

$$
2 x^{2}+(1-x)^{2}-(y-z)^{2} \leqslant 1
$$

Replacing $(1-x)$ by $(y+z)$, we have

$$
2 x^{2}+(y+z)^{2}-(y-z)^{2} \leqslant 1
$$

then $2 x^{2}+4 y z \leqslant 1$, which implies that

$$
x^{\prime}=x^{(2)}+2 y z \leqslant \frac{1}{2}
$$

Now, let us prove that if $0 \leqslant y \leqslant \frac{1}{2}$ then $0 \leqslant y^{\prime} \leqslant \frac{1}{2}$.

Consider $y^{\prime}=y^{2}+x-x^{2}$. Given that $0 \leqslant y \leqslant \frac{1}{2}, 0 \leqslant y^{2} \leqslant \frac{1}{4}$, the following is obtained:

$$
0 \leqslant x-x^{2} \leqslant \frac{1}{4}
$$

Therefore,

$$
0 \leqslant y^{\prime}=y^{2}+x-x^{2} \leqslant \frac{1}{2}
$$

Hence, $0 \leqslant y^{\prime} \leqslant \frac{1}{2}$.

Performing the same process for $z^{\prime}$ we have $0 \leqslant z^{\prime} \leqslant \frac{1}{2}$, thus, $A_{1}$ is an invariant region.

(2). By contrast, suppose that $A_{2}$ is invariant, which means that if $\frac{1}{2} \leqslant x \leqslant 1$, then $\frac{1}{2} \leqslant x^{(n)} \leqslant 1$. However,

$$
x^{\prime}=x^{2}+2 y z \leqslant x^{2}+2 x z=x(x+2 z),
$$

then $\frac{x^{\prime}}{x} \leqslant 1$. Therefore, $x^{(n)}$ decreasing sequence and bounded. Then $x^{(n)}$ converges and should go to a fixed point which means $\frac{1}{2} \leqslant x^{(n)} \leqslant 1$. However, this region does not have any fixed point, which contradicts our assumption. Hence, there is $n_{k} \in \mathbb{N}$ such that $x^{\left(n_{k}\right)}$ goes to the invariant region $A_{1} \cup A_{3}$, and never come back to $A_{2}$.

(3). This is proved by using the same process that was used to prove (2). Suppose that $A_{3}$ is invariant. Then, it follows that if $\frac{1}{2} \leqslant y \leqslant 1$. Then $\frac{1}{2} \leqslant y^{\prime} \leqslant 1$. It is easy to see that $y>x$, then one can easily check that the sequence $\left\{y^{(n)}\right\}$ is decreasing and bounded. Hence, $\left\{y^{(n)}\right\}$ will go to a fixed point, but no fixed point has this property in this region. Therefore, in both cases there is $n_{k} \in \mathbb{N}$ such that $x^{\left(n_{k}\right)}$ tends to the invariant region $A_{1}$, and this finding is precisely the assertion of the proposition.

Proposition 4.2. The following statements hold true for $\mathrm{V}_{\frac{1}{2}}$ :

(i) if $x^{(0)} \in B_{1}$, then there exists $n_{k} \in \mathbb{N}$ such that $\mathrm{V}^{\left(n_{k}\right)}(x) \in B_{2}$. Moreover, $B_{2}$ is invariant;

(ii) if $x^{(0)} \in \mathrm{B}_{3}$, then there exists $\mathrm{n}_{\mathrm{k}} \in \mathbb{N}$ such that $\mathrm{V}^{\left(\mathrm{n}_{\mathrm{k}}\right)}(\mathrm{x}) \in \mathrm{B}_{4}$. Moreover, $\mathrm{B}_{4} \cup \mathrm{B}_{5}$ is invariant;

(iii) if $x^{(0)} \in \mathrm{B}_{4}$, then there exists $\mathrm{n}_{\mathrm{k}} \in \mathbb{N}$ such that $\mathrm{V}^{\left(\mathrm{n}_{\mathrm{k}}\right)}(\mathrm{x}) \in \mathrm{B}_{5}$;

(iv) $\mathrm{B}_{5}$ is invariant.

Proof.

(i). By contrast, suppose that $B_{1}$ is invariant, which means that if $x \leqslant z$ then $x^{(n)} \leqslant z^{(n)}$. However,

$$
y^{2}-y+x-x^{2} \leqslant 0
$$

Therefore, the sequence $\left\{y^{(n)}\right\}$ is decreasing and bounded. Thus, it should go to fixed point, but $\frac{1}{3} \leqslant$ $y^{(n)} \leqslant \frac{1}{2}$. Hence, $y^{(n)} \rightarrow \frac{1}{3}$. Moreover, from Proposition 4.1, we have $y^{(n)}-z^{(n)}$ is decreasing sequence and bounded, then it will go to a fixed point. Therefore $y^{(n)}-z^{(n)} \rightarrow \frac{1}{3}$. Hence, $z^{(n)} \rightarrow 0$, which implies 
that $x^{(n)} \rightarrow \frac{2}{3}$. But $\left(\frac{2}{3}, \frac{1}{3}, 0\right)$ is not a fixed point, which follows that $n_{k}$ exists, such that $V^{\left(n_{k}\right)}(x)$ goes to $\mathrm{B}_{2} \cup \mathrm{B}_{3} \subset \mathrm{B}_{2}$.

Now, let us consider $x^{\prime}-z^{\prime}=x^{2}+2 y z-z^{2}-x+x^{2}$ and $x^{\prime}-z^{\prime}=2 x^{2}+2 y z-z^{2}-x$. One can easily check that the minimum value of $x^{\prime}-z^{\prime}$ occurs when $x^{(0)}=\left(\frac{1}{3}, \frac{1}{3}, \frac{1}{3}\right)$, and the minimum value is zero. Therefore $x^{\prime}>z^{\prime}$ whenever $x>z$. Thus, $B_{2}$ is invariant.

(ii). Suppose the assertion is false. Then $B_{3}$ is invariant, and it follows that

$$
0 \leqslant z^{(n)} \leqslant y^{(n)} \leqslant x^{(n)} \leqslant \frac{1}{2}
$$

Clearly, the sequence $\left\{z^{(n)}\right\}$ is decreasing and bounded in this region. Hence, the sequence is convergent and goes to a fixed point. However, no fixed point has this property in this region, which contradicts our expected findings. Therefore, there exists $n_{k} \in \mathbb{N}$ such that $V^{\left(n_{k}\right)}(x) \rightarrow B_{4}$. Now, we are going to show that $B_{4} \cup B_{5}$ is invariant. It follows from finding the minimum value of $f(x, y)=y^{\prime}-x^{\prime}=$ $y^{2}+x-x^{2}-x^{2}-2 y(1-x-y)$ on $B_{4} \cup B_{5}$ that the minimum value of $f$ is zero. Hence $y^{(n)}>x^{(n)}$, and $\mathrm{B}_{4} \cup \mathrm{B}_{5}$ is invariant.

(iii). Suppose contrary to our claim that $B_{4}$ is invariant then $x^{(n)} \geqslant \frac{1}{2}$ for all $n \in \mathbb{N}$. Thus, the sequence $\left\{z^{(n)}\right\}$ is decreasing and bounded, therefore, it is convergent and goes to fixed point 0 . Clearly, the sequence $\left\{y^{(n)}\right\}$ is a bounded and increasing sequence. Hence, $y^{(n)} \rightarrow \frac{1}{3}$, which means that $x^{(n)} \rightarrow \frac{2}{3}$ but $\left(\frac{2}{3}, \frac{1}{3}, 0\right)$ is not a fixed point. Thus, $n_{k} \in N$ exists such that $V^{\left(n_{k}\right)}(x)$ goes to $B_{5}$.

(iv). Let $x \leqslant \frac{1}{3}$. Then, $x^{\prime}=x^{2}+2 y z$ has a maximum value when $x^{(0)}=\left(\frac{1}{3}, \frac{1}{3}, \frac{1}{3}\right)$. Therefore, $x^{\prime} \leqslant \frac{1}{3}$, it follows that $x \leqslant \frac{1}{3}$. Thus, $x^{(n)} \leqslant \frac{1}{3}$. Using $x \geqslant z$ we have $x^{(n)} \geqslant z^{(n)} \geqslant 0$ because $y^{(n)}+x^{(n)}+z^{(n)}=1$, then we obtain $y^{(n)} \geqslant \frac{1}{3} \geqslant x^{(n)}$ and we conclude that $B_{5}$ is invariant, this completes the proof.

Theorem 4.3. Let $x^{(0)}=(x, y, z) \notin \operatorname{Fix}\left(V_{\frac{1}{2}}\right)$, then $\omega_{V_{\frac{1}{2}}}\left(x^{(0)}\right)=\left(\frac{1}{3}, \frac{1}{3}, \frac{1}{3}\right)$.

Proof. From Propositions 4.1 and 4.2, we only need to study the behavior of $V_{\frac{1}{2}}$ over $B_{5}$. We know that $y^{\prime}=y^{2}+x(1-x)$. Then, we obtain $y^{\prime} \leqslant y(y+2 x)$. Therefore, the sequence $\left\{y^{(n)}\right\}$ is bounded and decreasing; thus it should go to a fixed point. Hence, we have $y^{(n)} \rightarrow \frac{1}{3}$. However, the sequence $\left|y^{(n)}-z^{(n)}\right| \rightarrow 0$. Hence, $z^{(n)} \rightarrow \frac{1}{3}$. Using $x^{(n)}=1-y^{(n)}-z^{(n)}$ we obtain $x^{(n)} \rightarrow \frac{1}{3}$, which is the desired conclusion.

\section{Local behavior of $V_{a}$}

In this section, we will study the local behavior of $V_{a}$ around each fixed point through linearization around each fixed point of $V_{a}$ where $0<a<1$.

Following [3], suppose that the dynamical system has a fixed point $\bar{x}=\left(x^{*}, y^{*}, z^{*}\right)$, namely, there exists $\bar{x} \in S^{2}$ such that $\bar{x}=\phi(\bar{x})$. A Taylor expansion of $x_{i t+1}=\phi\left(x_{t}\right)$ around the fixed point $\bar{x}$, obtains

$$
x_{i t+1}=\phi^{i}\left(x_{t}\right)=\phi^{i}(\bar{x})+\sum_{j=1}^{n} \phi_{j}^{i}(\bar{x})\left(x_{j} t-\overline{x_{j}}\right)+\cdots+R_{n},
$$

where $\phi_{j}^{i}(\bar{x})$ is the partial derivatives of the function $\phi^{i}\left(x_{t}\right)$ with respect to $x_{j t}$ evaluated at $\bar{x}$, i.e, $\phi_{j}^{i}(\bar{x})=$ $\frac{\partial \phi^{i}\left(x_{t}\right)}{\partial x_{i t}}$. Thus, the linearized equation around the fixed point $\bar{x}$ is given by

$$
x_{i t+1}=\phi_{1}^{i}(\bar{x}) x_{1 t}+\phi_{2}^{i}(\bar{x}) x_{2 t}+\cdots+\phi_{n}^{i}(\bar{x}) x_{n t}+\phi^{i}(\bar{x})-\sum_{j=1}^{n} \phi_{j}^{i}(\bar{x}) \overline{x_{j}} .
$$

The linearized system, is therefore:

$$
\left[\begin{array}{c}
x_{1 t+1} \\
x_{2 t+1} \\
\vdots \\
x_{n t+1}
\end{array}\right]=\left[\begin{array}{cccc}
\phi_{1}^{1}(\bar{x}) & \phi_{2}^{1}(\bar{x}) & \cdots & \phi_{n}^{1}(\bar{x}) \\
\phi_{1}^{2}(\bar{x}) & \phi_{2}^{2}(\bar{x}) & \cdots & \phi_{n}^{2}(\bar{x}) \\
\vdots & & & \\
\phi_{1}^{n}(\bar{x}) & \phi_{2}^{n}(\bar{x}) & \cdots & \phi_{n}^{n}(\bar{x})
\end{array}\right]\left[\begin{array}{c}
x_{1 t} \\
x_{2 t} \\
\vdots \\
x_{n t}
\end{array}\right]+\left[\begin{array}{c}
\phi^{1}(\bar{x})-\sum_{j=1}^{n} \phi_{j}^{1}(\bar{x}) \overline{x_{j}} \\
\phi^{2}(\bar{x})-\sum_{j=1}^{n} \phi_{j}^{2}(\bar{x}) \overline{x_{j}} \\
\vdots \\
\phi^{n}(\bar{x})-\sum_{j=1}^{n} \phi_{j}^{n}(\bar{x}) \bar{x}_{j}
\end{array}\right] .
$$


Thus, the nonlinear system has been approximated locally by a linear system

$$
x_{t+1}=A x_{t}+B
$$

where

$$
A \equiv\left[\begin{array}{ccc}
\phi_{1}^{1}(\bar{x}) & \cdots & \phi_{\mathfrak{n}}^{1}(\bar{x}) \\
\phi_{1}^{2}(\bar{x}) & \cdots & \phi_{\mathfrak{n}}^{2}(\bar{x}) \\
\vdots & \ddots & \\
\phi_{1}^{\mathfrak{n}}(\bar{x}) & \cdots & \phi_{\mathfrak{n}}^{\mathfrak{n}}(\bar{x})
\end{array}\right] \equiv D \phi(\bar{x})
$$

is the Jacobian matrix of $\phi\left(x_{t}\right)$ evaluated at $\bar{x}$, and

$$
B \equiv\left[\begin{array}{c}
\phi^{1}(\bar{x})-\sum_{j=1}^{n} \phi_{j}^{1}(\bar{x}) \overline{x_{j}} \\
\phi^{2}(\bar{x})-\sum_{j=1}^{n} \phi_{j}^{2}(\bar{x}) \overline{x_{j}} \\
\vdots \\
\phi^{n}(\bar{x})-\sum_{j=1}^{n} \phi_{j}^{n}(\bar{x}) \overline{x_{j}}
\end{array}\right] .
$$

Theorem 5.1. Let $\mathrm{V}_{\mathrm{a}}: \mathrm{S}^{2} \rightarrow \mathrm{S}^{2}$ be a $\xi^{(s)}-Q S O$ given by (3.2). Then the following statements hold true.

(i) The local behavior of $\mathrm{V}_{\mathrm{a}}$ is stable around the following fixed points

$$
\begin{cases}\left(\frac{1}{3} \frac{1}{3}, \frac{1}{3}\right) & : a=\frac{1}{2}, \\ \left(x^{*}, y^{*}, z^{*}\right) & : a \in\left(\frac{1}{4}, \frac{1}{2}\right) \cup\left(\frac{1}{2}, \frac{3}{4}\right), \\ \left\{e_{3}\right\} & : a \in\left(0, \frac{1}{4}\right], \\ \left\{e_{2}\right\} & : a \in\left[\frac{3}{4}, 1\right) .\end{cases}
$$

(ii) If $\mathrm{a}=\frac{1}{2}$, then, the local behavior $\mathrm{V}_{\mathrm{a}}$ is unstable around $\left\{\mathrm{e}_{1}, \mathrm{e}_{2}, \mathrm{e}_{3}\right\}$.

(iii) If $\mathrm{a} \in\left(0, \frac{1}{4}\right)$, then, the local behavior $\mathrm{V}_{\mathrm{a}}$ is unstable around $\left\{\mathrm{e}_{2}\right\}$.

(iv) If $\mathrm{a} \in\left(\frac{3}{4}, 1\right)$, then, the local behavior $\mathrm{V}_{\mathrm{a}}$ is unstable around $\left\{\mathrm{e}_{3}\right\}$.

Proof.

(i). Let $a=\frac{1}{2}$. Then, we linearize $V_{a}$ around the fixed points when $a=\frac{1}{2}$. Thus the linearization of $V_{\frac{1}{2}}$ around the fixed point $\left(\frac{1}{3}, \frac{1}{3}, \frac{1}{3}\right)$ has the following form:

$$
\left\{\begin{array}{l}
x^{\prime}=\frac{2}{3} x+\frac{2}{3} y+\frac{2}{3} z-\frac{1}{3} \\
y^{\prime}=\frac{1}{3} x+\frac{2}{3} y \\
z^{\prime}=\frac{1}{3} x+\frac{2}{3} z
\end{array}\right.
$$

We can rewrite first equation of system (5.1) as $\frac{2}{3}(x+y+z)-\frac{1}{3}=\frac{1}{3}$. It follows that $x^{(n)}$ goes to $\frac{1}{3}$. Therefore, by induction of equation (2) in (5.1) we obtain $y^{(n)}=\left(\frac{2}{3}\right)^{n} y+\frac{1}{9} \sum_{k=1}^{n}\left(\frac{2}{3}\right)^{k-1}$. Thus, the limit of the last expression is taking into account that the sum of convergent geometric series is $\frac{1}{3}$. Hence $\lim _{n \rightarrow \infty} y^{(n)}=\frac{1}{3}$. Consequently, $z^{(n)}=1-x^{(n)}+y^{(n)}=\frac{1}{3}$. Hence, $V_{\frac{1}{2}}$ is stable around $\left(\frac{1}{3}, \frac{1}{3}, \frac{1}{3}\right)$.

Moreover, let $a \in\left(\frac{1}{4}, \frac{1}{2}\right) \cup\left(\frac{1}{2}, \frac{3}{4}\right)$. Then, we can rewrite the linearization of $V_{a}$ around $\left(x^{*}, y^{*}, z^{*}\right)$ as follows:

$$
\left\{\begin{array}{l}
x^{\prime}=\left(2 x^{*}-2 y^{*}\right) x+\left(2 z^{*}-2 y^{*}\right) y+2 y^{*}+x^{*}-2\left(x^{*}\right)^{2}-4 y^{*} x^{*} \\
y^{\prime}=2 a\left(1-2 x^{*}\right) x+2 y^{*} y+y^{*}-2 a\left(1-2 x^{*}\right)-2\left(y^{*}\right)^{2}
\end{array}\right.
$$

Let us define the following matrix

$$
\tilde{A}=\left(\begin{array}{cc}
2 x^{*}-2 y^{*} & 2 z^{*}-2 y^{*} \\
2 a\left(1-2 x^{*}\right) & 2 y^{*}
\end{array}\right)
$$


by using $\tilde{\mathrm{A}}=\mathrm{QDQ}^{-1}$ where $\mathrm{D}$ is a diagonal matrix given as follows:

$$
\tilde{A}=\left(\begin{array}{cc}
f(a) & 0 \\
0 & g(a)
\end{array}\right),
$$

where $f(a)=\frac{16 a^{2}-16 a+3+\sqrt{1+64 a-320 a^{2}+512 a^{3}-256 a^{4}}}{16 a^{2}-16 a+1}$ and $g(a)=\frac{-3+16 a-16 a^{2}+\sqrt{1+64 a-320 a^{2}+512 a^{3}-256 a^{4}}}{16 a^{2}-16 a+1}$. One can easily check $0<|\mathrm{f}(\mathrm{a})|<1$ and $0<|\mathrm{g}(\mathrm{a})|<1$. However,

$$
x^{(n)}=Q^{n} Q^{-1}(x-\bar{x})+\bar{x} .
$$

Then, $\lim _{n \rightarrow \infty} x^{(n)}=\bar{x}$ where $\bar{x}=\left(x^{*}, y^{*}, z^{*}\right)$. Therefore, $V_{a}$ is stable around $\left(x^{*}, y^{*}, z^{*}\right)$.

We now look at the stability of $V_{a}$ around $e_{2}$ and $e_{3} . V_{a}$ is linearized around $e_{3}$. Then we have

$$
\left\{\begin{array}{l}
x^{\prime}=2 y \\
y^{\prime}=2 a x, \\
z^{\prime}=2(1-a) x+2 z-1
\end{array}\right.
$$

After induction of the first equation of (5.2), we obtain the sub-sequences $x^{(2 n)}=(4 a)^{n} x, x^{(2 n+1)}=$ $(4 a)^{n} 2 y, y^{(2 n)}=(4 a)^{n} y$ and $y^{(2 n-1)}=(4 a)^{n} \frac{x}{2}$. It is easy to see that the sequences are convergent when $a \in\left(0, \frac{1}{4}\right]$ and go to zero, so, in general $x^{n}, y^{n}$ converge to zero, therefore, $z^{n}$ converges to one. Hence, the behavior of $V_{a}$ around $e_{3}$ is stable when $a \in\left(0, \frac{1}{4}\right]$. Finally, the linearization of $V_{a}$ around $e_{2}$ has the following form:

$$
\left\{\begin{array}{l}
x^{\prime}=2 z, \\
y^{\prime}=2 a x+2 y-1, \\
z^{\prime}=2(1-a) x .
\end{array}\right.
$$

From the induction of the first and third equations of the system (5.3) we obtain the following subsequences: $x^{(2 n)}=2^{(2 n)}(1-a)^{n} x, x^{(2 n+1)}=2^{(2 n+1)}(1-a)^{n} z, z^{(2 n)}=2^{(2 n)}(1-a) z$ and $z^{(2 n-1)}=$ $2^{(2 n-1)}(1-a) x$. One can easily show that if $a \in\left[\frac{3}{4}, 1\right)$, then the sequences $x^{n}, z^{n}$ are convergent and go to zero. Therefore, the sequence $z^{n}$ is convergent and goes to one.

The sequences are clearly divergent when $a \in\left(\frac{1}{4}, \frac{1}{2}\right) \cup\left(\frac{1}{2}, \frac{3}{4}\right)$. Hence, the behavior of $V_{a}$ around $e_{3}$ is unstable when $a \in\left(\frac{1}{4}, \frac{1}{2}\right) \cup\left(\frac{1}{2}, \frac{3}{4}\right)$.

(ii). Now, let us look at the stability of $V_{\frac{1}{2}}$ around $e_{1}$. The linearization of $V_{\frac{1}{2}}$ takes the following form

$$
\left\{\begin{array}{l}
x^{\prime}=2 x-1 \\
y^{\prime}=-x+1 \\
z^{\prime}=-x+1
\end{array}\right.
$$

On the basis of second and third equations of system (5.4), we obtain $z^{(n)}=y^{(n)}=2^{n-1}(1-x)$, which is a divergent sequence. Therefore, $V_{\frac{1}{2}}$ is unstable around $e_{1}$. We now study the local behavior of $V_{\frac{1}{2}}$ around $e_{2}$. The linearization of $V_{\frac{1}{2}}$ around $e_{2}$ is

$$
\left\{\begin{array}{l}
x^{\prime}=2 x-1, \\
y^{\prime}=-x+1, \\
z^{\prime}=-x+1
\end{array}\right.
$$

Here, we consider two subsequences $x^{(2 n)}=2^{n} x$ and $x^{(2 n-1)}=2^{n} x$, which are both divergent. Thus, $V_{\frac{1}{2}}$ is unstable around $e_{2}$. In the same manner, we can see that $V_{\frac{1}{2}}$ is unstable around $e_{3}$.

(iii). From proof of (i), we can easily see that the induction of linearization around $e_{2}$ gives the following subsequences: $x^{(2 n)}=2^{(2 n)}(1-a)^{n} x, x^{(2 n+1)}=2^{(2 n+1)}(1-a)^{n} z, z^{(2 n)}=2^{(2 n)}(1-a) z$, and $z^{(2 n-1)}=$ $2^{(2 n-1)}(1-a) x$. Clearly if $a \in\left(0, \frac{1}{4}\right)$, then $x^{n}$ and $z^{n}$ are divergent. Hence, the local behavior of $V_{a}$ is unstable around $e_{2}$.

(iv). From proof of (i), we can easily see that the induction of linearization around $e_{3}$ gives the following subsequences: $x^{(2 n)}=(4 a)^{n} x, x^{(2 n+1)}=(4 a)^{n} 2 y, y^{(2 n)}=(4 a)^{n} y$ and $y^{(2 n-1)}=(4 a)^{n} \frac{x}{2}$. Clearly if $a \in\left(\frac{3}{4}, 1\right)$, then $x^{n}$ and $y^{n}$ are divergent. Hence, the local behavior of $V_{a}$ is unstable around $e_{3}$. 


\section{Acknowledgment}

The authors are grateful to Prof. Farrukh Mukhamedov for his valuable comments and remarks, that improved the presentation of this paper.

\section{References}

[1] S. Bernstein, Solution of a mathematical problem connected with the theory of heredity, Ann. Math. Statistics, 13 (1942), 53-61. 1

[2] A. Dohtani, Occurrence of chaos in higher-dimensional discrete-time systems, SIAM J. Appl. Math., 52 (1992), 17071721. 2

[3] O. Galor, Discrete dynamical systems, Springer, Berlin, (2007). 2.4, 5

[4] R. N. Ganikhodzhaev, A family of quadratic stochastic operators that act in $\mathrm{S}^{2}$, (Russian); Dokl. Akad. Nauk UzSSR, 1 (1989), 3-5. 1

[5] R. N. Ganikhodzhaev, Quadratic stochastic operators, Lyapunov functions and tournaments, (Russian); translated from Mat. Sb., 183 (1992), 119-140, Russian Acad. Sci. Sb. Math., 76 (1992), 489-506. 1, 2, 2.2

[6] R. N. Ganikhodzhaev, A chart of fixed points and Lyapunov functions for a class of discrete dynamical systems, (Russian); translated from Mat. Zametki, 56 (1994), 40-49, Math. Notes, 56 (1994), 1125-1131. 1, 2

[7] N. N. Ganikhodzhaev, An application of the theory of Gibbs distributions to mathematical genetics, Doklady Math., 61 (2000), 321-323. 1

[8] R. N. Ganikhodzhaev, R. É. Abdirakhmanova, Description of quadratic automorphisms of a finite-dimensional simplex, (Russian); Uzbek. Mat. Zh., 1 (2002), 7-16. 1

[9] R. N. Ganikhodzhaev, A. M. Dzhurabaev, The set of equilibrium states of quadratic stochastic operators of type $V_{\pi}$, (Russian); Uzbek. Mat. Zh., 3 (1998), 23-27. 1

[10] R. N. Ganikhodzhaev, D. B. Éshmamatova, Quadratic automorphisms of a simplex and the asymptotic behavior of their trajectories, (Russian); Vladikavkaz. Mat. Zh., 8 (2006), 12-28. 1, 2

[11] R. Ganikhodzhaev, F. Mukhamedov, U. Rozikov, Quadratic stochastic operators and processes: results and open problems, Infin. Dimens. Anal. Quantum Probab. Relat. Top., 14 (2011), 270-335. 1

[12] N. N. Ganikhodzhaev, R. T. Mukhitdinov, On a class of measures corresponding to quadratic operators, (Russian); Dokl. Akad. Nauk Rep. Uzb., 3 (1995), 3-6. 1

[13] N. N. Ganikhodzhaev, U. A. Rozikov, On quadratic stochastic operators generated by Gibbs distributions, Regul. Chaotic Dyn., 11 (2006), 467-473. 1

[14] J. Hofbauer, V. Hutson, W. Jansen, Coexistence for systems governed by difference equations of Lotka-Volterra type, J. Math. Biol., 25 (1987), 553-570. 1, 2

[15] J. Hofbauer, K. Sigmund, The theory of evolution and dynamical systems, Mathematical aspects of selection, Translated from the German, London Mathematical Society Student Texts, Cambridge University Press, Cambridge, (1988). 1,2

[16] R. D. Jenks, Quadratic differential systems for interactive population models, J. Differential Equations, 5 (1969), 497-514. 1,2

[17] H. Kesten, Quadratic transformations: A model for population growth, II, Advances in Appl. Probability, 2 (1970), 179-228. 1

[18] S.-T. Li, D.-M. Li, G.-K. Qu, On stability and chaos of discrete population model for a single-species with harvesting, J. Harbin Univ. Sci. Tech., 6 (2006), 021. 1

[19] A. J. Lotka, Undamped oscillations derived from the law of mass action, J. Amer. Chem. Soc., 42 (1920), 1595-1599. 2

[20] Y. I. Lyubich, Mathematical structures in population genetics, Translated from the 1983 Russian original by D. Vulis and A. Karpov, Biomathematics, Springer-Verlag, Berlin, (1992). 1

[21] F. Mukhamedov, A. H. M. Jamal, On $\xi^{\text {s }-q u a d r a t i c ~ s t o c h a s t i c ~ o p e r a t o r s ~ i n ~ 2-d i m e n s i o n a l ~ s i m p l e x, ~ P r o c . ~ o f ~ t h e ~ 6 t h ~ I M T-~}$ GT Inter. Conf. on Math., Stat. and Its Appl., Universiti Tunku Abdul Rahman, Kuala Lumpur, Malaysia, (2010), 159-172. 1

[22] F. Mukhamedov, M. Saburov, A. H. M. Jamal, On dynamics of $\xi^{s}$-quadratic stochastic operators, Inter. Jour. Modern Phys., Conf. Ser., 9 (2012), 299-307. 1

[23] F. Mukhamedov, M. Saburov, I. Qaralleh, Classification of $\left.\xi^{(} s\right)$-quadratic stochastic operators on 2D simplex, J. Phys., Conf. Ser., 435 (2013), 8 pages.

[24] F. Mukhamedov, M. Saburov, I. Qaralleh, On $\xi^{(s)}$-quadratic stochastic operators on two-dimensional simplex and their behavior, Abstr. Appl. Anal., 2013 (2013), 12 pages. 1, 2, 2.3, 3

[25] M. Plank, Hamiltonian structures for the n-dimensional Lotka-Volterra equations, J. Math. Phys., 36 (1995), $3520-3543$. 1,2

[26] U. A. Rozikov, N. B. Shamsiddinov, On non-Volterra quadratic stochastic operators generated by a product measure, Stoch. Anal. Appl., 27 (2009), 353-362. 1

[27] U. A. Rozikov, A. Zada, On dynamics of $\ell$-Volterra quadratic stochastic operators, Int. J. Biomath., 3 (2010), 143-159. 1, $2,2.2$ 


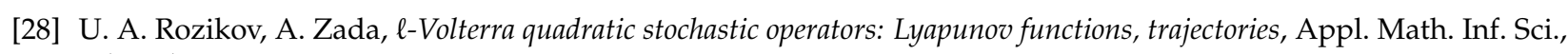
6 (2012), 329-335. 1, 2

[29] U. A. Rozikov, U. U. Zhamilov, F-quadratic stochastic operators, (Russian); translated from Mat. Zametki, 83 (2008), 606-612, Math. Notes, 83 (2008), 554-559. 1

[30] M. Saburov, Some strange properties of quadratic stochastic Volterra operators, World Appl. Sci. J., 21 (2013), 94-97. 1

[31] P. R. Stein, S. M. Ulam, Non-linear transformation studies on electronic computers, Rozprawy Mat., 39 (1964), 66 pages. 1

[32] F. E. Udwadia, N. Raju, Some global properties of a pair of coupled maps: quasi-symmetry, periodicity, and synchronicity, Phys. D, 111 (1998), 16-26. 1

[33] S. M. Ulam, Problems in modern mathematics, Science Editions John Wiley \& Sons, Inc., New York, (1964). 1, 2

[34] V. Volterra, Lois de fluctuation de la population de plusieurs espèces coexistant dans le même milieu, Association Franc. Lyon., 1926 (1927), 96-98. 1, 2

[35] M. I. Zaharevič, The behavior of trajectories and the ergodic hypothesis for quadratic mappings of a simplex, (Russian); Uspekhi Mat. Nauk, 33 (1978), 207-208. 1

[36] U. U. Zhamilov, U. A. Rozikov, On the dynamics of strictly non-Volterra quadratic stochastic operators on a twodimensional simplex, (Russian); translated from Mat. Sb., 200 (2009), 81-94, Sb. Math., 200 (2009), 1339-1351. 1 